\title{
Liderazgo y gestión estratégica en sistemas de salud basados en atención primaria de salud
}

\author{
Eduardo Benjamin Puertas ${ }^{1}$, Juan Manuel Sotelo ${ }^{1}$ y Gilma Ramos ${ }^{2}$
}

Forma de citar

Puertas EB, Sotelo JM, Ramos G. Liderazgo y gestión estratégica en sistemas de salud basados en atención primaria de salud. Rev Panam Salud Publica. 2020;44:e124. https://doi.org/10.26633/RPSP.2020.124

RESUMEN

Objetivos. Identificar y analizar varios tipos de liderazgo y de gestión que podrían aportar al fortalecimiento de los sistemas de salud basados en atención primaria en salud (APS).

Métodos. Se realizó una revisión estructurada de literatura en gestión y liderazgo aplicable a los sistemas de salud basados en APS. De los 19 artículos identificados se extrajeron detalles relevantes usando una abstracción estandarizada.

Resultados. Los sistemas de salud basados en APS deben establecer prácticas de gestión óptimas para alcanzar la calidad, la eficiencia y la efectividad. La planeación y gestión estratégica facilitan la formación de una identidad común, aunque existen organizaciones sobregerenciadas y pobremente lideradas. La salud universal es un impulso estratégico que requiere de un liderazgo transformador para avanzar hacia sistemas basados en APS. El liderazgo en ellos debe centrarse en identificar los desafíos adaptativos, vincular al personal y proteger el liderazgo de las bases, al igual que debe adaptarse a los momentos y combinar las lógicas de acción del estratega para lograr una transformación sectorial. Los modelos de calidad establecen criterios de liderazgo similares a otras teorías, destacando como aportes la innovación y la identidad organizacional. Conclusiones. América Latina debe encaminarse hacia sistemas de salud basados en APS participativos, con líderes con inteligencia emocional, que descubran sus propios eventos transformadores, busquen la calidad y combinen varios tipos de liderazgo. Deben ser estrategas para liderar al sistema en su transición hacia organizaciones resilientes, y "alquimistas" para lograr verdaderas transformaciones en salud, con la voluntad y humildad de quien trabaja para alcanzar la salud universal.

Palabras claves Gestión en salud; liderazgo; sistemas de salud; atención primaria de salud; Américas.

La atención primaria de salud (APS) es concebida como "una estrategia integral para intervenir en los determinantes sociales y abrir espacios concretos para que las comunidades sean partícipes de los modelos de atención del siglo XXI". ${ }^{i} \mathrm{La}$ propuesta APS 30-30-30 de un nuevo Pacto Regional por la Atención Primaria de Salud para la Salud Universal por parte de la Organización Panamericana de la Salud (OPS) en respuesta al Informe "Salud Universal en el Siglo XXI: 40 años de

Michelle Bachelet en la Presentación del Informe de la Comisión de Alto Nivel "Salud Universal en el Siglo XXI: 40 años de Alma-Ata".
Alma-Ata" (1), marca metas que buscan la disminución en 30\% de las barreras que dificultan el acceso a la salud y destinar $30 \%$ del total de presupuesto público en salud al nivel primario, para el año 2030.

Un sistema de salud es "el conjunto de todas las organizaciones públicas y privadas, instituciones y recursos que intervienen para mejorar, mantener o restaurar la salud" (2). Los sistemas de salud "abarcan tanto los servicios, a la persona como a la población, así como las actividades para influir en las políticas y acciones de otros sectores para abordar los determinantes sociales, ambientales y económicos de la salud" (2). Un sistema

\footnotetext{
1 Organización Panamericana de la Salud, San Salvador, El Salvador $₫$ Eduardo Benjamin Puertas, puertasb@paho.org
} 
de salud basado en la atención primaria de salud (SS-APS) es "una estrategia de organización integral cuya meta principal es alcanzar el derecho universal a la salud con la mayor equidad y solidaridad y que se basa en los principios de la APS, como el compromiso de dar respuesta a las necesidades de salud de la población, la orientación a la calidad, la responsabilidad y la rendición de cuentas de los gobiernos, la justicia social, la sostenibilidad, la participación y la intersectorialidad y debe prestar atención integral, integrada y apropiada a lo largo del tiempo, hacer hincapié en la prevención y en la promoción y garantizar la atención del paciente en el primer contacto" (3).

Las iniciativas para el fortalecimiento y cambio del modelo de atención, y para la organización de los servicios de salud con una perspectiva de APS integral, han enfrentado serios desafíos para su implementación $(4,5)$. Una concepción de APS integral y el sostenimiento en el tiempo de esfuerzos para fortalecer el primer nivel de atención podrían ser factores facilitadores de las mejoras en las condiciones de acceso (6). Para esto se requiere un liderazgo comprometido y sostenido que permita avanzar hacia sistemas de salud basados en APS. El liderazgo es necesario para desarrollar intervenciones y construir alianzas intersectoriales sostenibles que operen en diferentes sectores y esferas institucionales del sistema de salud. Para abordar los desafíos en salud las autoridades nacionales de salud requieren el fortalecimiento de su capacidad de liderazgo, planificación, regulación y negociación.

El artículo tiene como objetivo analizar varios tipos de liderazgo y de gestión que podrían aportar al fortalecimiento de sistemas de salud basados en APS en su avance hacia la salud universal.

\section{MATERIALES Y MÉTODOS}

Se realizó una revisión estructurada en gestión y liderazgo en SS-APS. Para la estrategia de búsqueda los autores revisaron la literatura indexada utilizando términos generales para maximizar la sensitividad, seguida de la utilización más refinada de términos de búsqueda. Las palabras claves fueron ajustadas a los Descriptores de Ciencias de la Salud y se utilizaron los equivalentes en idioma español e idioma inglés, de acuerdo al cuadro 1.

CUADRO 1. Términos de búsqueda y palabras clave de la revisión estructurada

\begin{tabular}{|c|c|c|}
\hline Palabras clave (inglés) & $\begin{array}{l}\text { Palabras clave } \\
\text { (español) }\end{array}$ & Términos de búsqueda \\
\hline Health management & Gestión en salud & $\begin{array}{l}\text { Health management AND health } \\
\text { systems } \\
\text { Health management AND primary } \\
\text { health care }\end{array}$ \\
\hline Leadership & Liderazgo & $\begin{array}{l}\text { Leadership AND health systems } \\
\text { Leadership AND primary health } \\
\text { care }\end{array}$ \\
\hline Health systems & Sistemas de Salud & $\begin{array}{l}\text { Health systems AND primary } \\
\text { health care }\end{array}$ \\
\hline Primary health care & $\begin{array}{l}\text { Atención primaria } \\
\text { de salud }\end{array}$ & \\
\hline PHC based health systems & $\begin{array}{l}\text { Sistemas de salud } \\
\text { basados en APS }\end{array}$ & \\
\hline
\end{tabular}

Fuente: los autores

PHC, primary health care
Se usaron las bases de datos de PubMed y Google Scholar. Para incluirlos en el estudio, los artículos debían abordar teorías o características de liderazgo y gestión que sean aplicables al contexto de sistemas de salud. De 35 artículos revisados se seleccionaron 19 que cumplían los criterios señalados. Los autores extrajeron detalles relevantes de los artículos usando una abstracción estandarizada que incluyó los principales hallazgos y observaciones.

Los autores utilizaron métodos de síntesis narrativa para integrar los hallazgos en resúmenes descriptivos usando el proceso de cuatro elementos de Popay (7): 1) Aportar con insumos para el desarrollo de una teoría sobre el tipo de gestión y liderazgo para sistemas de salud basados en APS; 2) desarrollar una síntesis preliminar; 3) explorar relaciones dentro y entre los diferentes estudios; y, 4) valorar la robustez de la síntesis.

\section{RESULTADOS}

\section{Gestión estratégica en sistemas de salud basados en APS}

La gestión se refiere a enfrentar la complejidad y la incertidumbre, introduciendo orden y predictibilidad a una situación, y como sostiene Kotter (8), entendiendo que para desarrollarse las organizaciones deben adaptarse al cambio. Ginter y cols (9) establecen tres etapas de la gestión estratégica: el pensamiento estratégico, la planificación estratégica y la gestión del impulso estratégico. El pensamiento estratégico es aquel que permite mirar el panorama general o "desde el balcón" como lo afirman Heifetz y Laurie (10). La planificación estratégica involucra un análisis del entorno externo, un análisis del entorno interno y el desarrollo o refinamiento de las estrategias direccionales organizacionales. El impulso se refiere a aprovechar el momentum para implementar la planificación estratégica.

La gestión estratégica tiene dos enfoques: el enfoque analítico y el enfoque emergente. El enfoque analítico o racional depende del desarrollo de una secuencia lógica de pasos o procesos, mientras que el modelo emergente depende del pensamiento intuitivo, del liderazgo y del aprendizaje. El enfoque analítico se le podría comparar con un mapa que detalla un mundo conocido, mientras que el emergente se compara con una brújula que da un sentido de dirección cuando se atraviesa territorios desconocidos (9).

Los sistemas de salud en la Región de las Américas se enfrentan a un entorno cada vez más turbulento, confuso y amenazador. Dichos cambios se relacionan con el marco legal y políticas públicas; la influencia de las fuerzas económicas y de mercado a nivel nacional e internacional; la transición demográfica y cambios en los estilos de vida; avances tecnológicos; $\mathrm{y}$, cambios fundamentales en la atención de salud. (9) Los cambios más dramáticos en las organizaciones de atención de la salud comenzaron en la década de los ochenta y continúan en nuestros días, pero la realidad es que todavía son "novicias para trabajar en entornos altamente volátiles y competitivos, que otras industrias han venido enfrentando durante años" (11).

En los sistemas de salud basados en APS se considera importante "establecer prácticas de gestión óptimas en todos los niveles para alcanzar la calidad, la eficiencia y la efectividad necesarias y para desarrollar mecanismos activos que eleven al máximo la participación individual y colectiva en la gestión de salud" (3). Al considerar la planificación en los sistemas de 
salud, la elaboración de política pública sanitaria es uno de los elementos fundamentales para la función de rectoría y gobernanza y el producto más visible de la planificación. Muchos de esos esfuerzos de planificación vinculados a políticas de salud siguen vinculados a programas para el control de enfermedades transmisibles y no transmisibles, y en general determinan las normas que rigen para prestadores y usuarios de la red de servicios de salud. Sin embargo, estos esfuerzos de planificación no representan una gestión estratégica.

Otro producto visible de la planificación es el plan de acción. Drucker afirma que el plan de acción es una declaración de intenciones más que un compromiso, que no debe volverse en una camisa de fuerza y que debe ser revisado a menudo, pues "cada éxito crea nuevas oportunidades, al igual que cada fracaso" (12). Kotter señala que existen muchas organizaciones sobregerenciadas y pobremente lideradas, uno de cuyos errores más frecuentes es "embarcarse en una planificación de largo plazo como una panacea para su falta de direccionamiento e inhabilidad para adaptarse a un entorno cada vez más dinámico y competitivo" (8).

Aunque existe amplia evidencia de la aplicación de la planificación y gestión estratégica en el sector público, no existe suficiente información sobre la extensión de uso de estas herramientas a nivel de entidades de gobierno y del sistema de salud, cómo son implementadas y los resultados que generan. Poister (13) concluye que existe evidencia empírica substancial del impacto de los determinantes ambientales, institucionales y organizacionales en la gestión estratégica de organizaciones públicas, pero "son escasos los esfuerzos para evaluar las relaciones entre los procesos de planificación estratégica y los resultados o las mejoras en el desempeño organizacional"(13). Nutt (14) diferencia la aplicación de decisiones estratégicas en el sector público y privado, encontrando diferencias que explican las dificultades en el nivel gubernamental (cuadro 2).

Harris (15) enfatiza la formación de "metaidentidades", organizaciones con identidades y prioridades diferentes. Los sistemas de salud están conformados por organizaciones públicas, autónomas y privadas, cada una con diferentes identidades y prioridades. En este escenario complejo, la planeación y gestión estratégica se convierten en el tejido conectivo que facilita la formación de una identidad compartida, transformando la conceptualización fragmentada de los principales actores, en un sistema integrado, global y con un propósito definido, como el que caracteriza al SS-APS. Harris lo llama "reconciliación de identidades organizacionales diferentes que permita la identificación de prioridades" (15). Al crear esta meta-identidad dentro de un sistema de salud fragmentado y segmentado se genera el mensaje de que el sistema se puede encargar de diferentes acciones, pero que lo hace de una forma constructiva y deliberada. Conforme el entorno se vuelve más complejo se requiere identificar cómo las multiplicidades de identidades coexisten dentro del sistema, cómo están construidas, reconstruidas, gerenciadas y lideradas. (15) En el mismo contexto, Kellermanns y cols. (16) señalan que el consenso estratégico está positivamente asociado con el desempeño, lo que corrobora la necesidad de un trabajo constructivo y colaborativo en el contexto de sistemas de salud que avanzan hacia la salud universal.

Se cuestiona a la intuición como una herramienta de toma de decisiones estratégicas. La evidencia no desconoce el papel que puede tener en concebir un direccionamiento guiado por un "sentido estratégico que trasciende la lógica ordinaria" (9). Miller (17) define dos tipos de intuición: presentimiento holístico y experiencia automatizada. En el primer caso, la apreciación o elección se hace a través de un proceso subconsciente que involucra la síntesis de experiencias variadas, combinación de información novedosa y sentimientos intensos de estar en lo correcto. En la intuición de experiencia automatizada la elección se hace a través de un proceso parcialmente subconsciente que involucra los pasos surgidos de experiencias pasadas en situaciones específicas, repeticiones de aprendizajes pasados y un sentimiento de familiaridad. La intuición es una herramienta de toma de decisiones problemática, que no tiene cabida en SS-APS, y que de usarse debe hacérselo de manera selectiva y cautelosa.

\section{Liderazgo en sistemas de salud basados en APS}

Liderazgo se refiere a los comportamientos y acciones que toma el líder para inspirar, convencer o impulsar al personal y a la organización hacia el logro de la visión (18). Los SS-APS requieren de un liderazgo dirigido a una transformación desde las bases de la atención primaria para alcanzar modelos más equitativos, eficientes y eficaces.

Los sistemas de salud generan desafíos adaptativos, que son problemas sistémicos poco claros y sin una respuesta fácil (10). Las soluciones se encuentran en la inteligencia colectiva de quienes conforman la organización a todo nivel, que en el caso de los sistemas de salud son las instituciones y actores. Este liderazgo debe centrarse en mirar toda la cancha, identificar los desafíos adaptativos, regular el distrés, vincular al personal y proteger un liderazgo que surja desde las bases. Heifetz cuestiona algunas de las perspectivas tradicionales sobre liderazgo, "...la noción prevalente de que el liderazgo consiste en tener una visión y en alienar personas con esa visión está en bancarrota, porque continúa abordando situaciones adaptativas como si fueran técnicas: presupone que la figura autoritaria debe adivinar hacia dónde va la organización, y se espera que la gente le siga" (10).

Kotter (8) sostiene que no se puede gestionar y liderar. Para avanzar hacia la salud universal, los SS-APS requieren de un

CUADRO 2. Aplicación de la planificación y gestión estratégica en el sector público y privado

\begin{tabular}{|c|c|}
\hline Público & Privado \\
\hline Favorece la colaboración entre organizaciones & $\checkmark$ Apuesta por la competencia y el lucro \\
\hline Datos de desempeño e información de inteligencia limitados & $\checkmark$ Datos de desempeño e información de inteligencia disponibles \\
\hline Mandatos y obligaciones limitan la autonomía y flexibilidad en mayor medida & $\checkmark$ Mandatos y obligaciones limitan la autonomía y flexibilidad en menor medida \\
\hline$\checkmark$ Mayor necesidad de consenso & $\checkmark$ Menor necesidad de consenso \\
\hline$\checkmark \quad$ Influencia política directa & $\checkmark$ Influencia política indirecta e interna, con frecuencia confidencial. \\
\hline$\checkmark$ Metas cambiantes & $\checkmark$ Metas claras y consensuadas \\
\hline
\end{tabular}

Fuente: elaborado por los autores con base en (14). 
liderazgo transformador, mientras que las instituciones que lo conforman requieren de una gestión estratégica sostenida que permita implementar los cambios necesarios identificados desde ese liderazgo. La realidad de nuestros sistemas de salud es que son gestionados en exceso y liderados pobremente. Esto no significa que el sistema de salud no requiere de gestión, ni que la organización no requiere de liderazgo. Kotter afirma que un ejército en tiempos de paz puede sobrevivir con una buena administración y gestión en todos los niveles jerárquicos, junto a un buen liderazgo concentrado en el tope del escalafón. Un ejército en tiempos de guerra necesita un liderazgo competente en todos los niveles, pues no se gestiona gente a la batalla, se lidera. (8) Es posible transitar entre la gestión y el liderazgo y viceversa, dependiendo de las circunstancias. Los sistemas de salud atraviesan por periodos de crisis y otros de calma relativa. Las crisis las desatan las emergencias sanitarias, el desabastecimiento de medicamentos e insumos, la falta de recursos humanos y financieros, las huelgas, entre otros. Desde esta perspectiva, en tiempos de calma relativa una adecuada administración y gestión del sistema y las entidades que lo conforman, con un buen liderazgo desde la autoridad sanitaria nacional, permitiría avanzar en las acciones hacia la salud universal. Sin embargo, este escenario es poco frecuente, pues a diferencia del ejército, el sector salud convive con situaciones de emergencia sanitaria, financiera, de personal y de logística que se suceden sin conceder una pausa. Los "tiempos de paz" son escasos, la crisis es habitual y el sistema de salud requiere de un liderazgo competente en todos los niveles. Aquí resuena la sentencia de Heifetz, para quien el "liderazgo debe ocurrir todos los días. No puede ser la responsabilidad de unos pocos, un evento raro, o una oportunidad en toda la vida" (10). Por ende, el liderazgo único y jerárquico no es suficiente, se requieren diferentes tipos de liderazgo que surjan desde todos los niveles del SS-APS.

Heifetz (10) señala que el trabajo del líder es "sacar a flote el conflicto y usarlo como una fuente de creatividad." Bennis (19) sostiene que los líderes extraordinarios encuentran significado y aprenden de los eventos más negativos a los que denomina "crucibles", experiencias significativas intensas, a menudo traumáticas, que producen un nuevo sentido de identidad y de las que el individuo se levanta más fuerte y confiado. Desde estos puntos de vista, Latinoamérica debería contar con una legión de líderes forjados en estas lides, pero la realidad no lo demuestra así. Las experiencias significativas a las que se refiere Bennis son del orden interno e implican otra dimensión más inherente al individuo que a las circunstancias de su entorno. Eso dificulta la identificación del líder para el sistema, que haya acumulado crucibles que le den la fortaleza y confianza para avanzar hacia la salud universal, que utilice el conflicto a su favor, que tenga la inteligencia emocional y empatía para lograr los cambios.

Collins (20) establece cinco niveles de jerarquía en liderazgo: Individuo altamente capaz (nivel 1), miembro de equipo que contribuye al alcance de los objetivos (nivel 2), gerente competente (nivel 3), líder efectivo (nivel 4) y ejecutivo (nivel 5). El liderazgo de nivel 5 es el que logra las transformaciones organizacionales a través de una combinación paradójica de humildad personal y voluntad profesional. Son líderes con una dualidad inherente: modestos y a la vez tenaces, tímidos pero intrépidos, que miran al balcón, no al espejo. Goleman posiciona a la inteligencia emocional, pues "...no solo distingue a los grandes líderes, sino que también puede ser relacionada con un desempeño excelente" (21). David Rooke (22) afirma que los grandes líderes se diferencian por sus acciones y define siete tipos de lógica de acción con sus fortalezas y debilidades: Oportunista, diplomático, experto, triunfador, individualista, estratega y alquimista, siendo estos dos últimos quienes alcanzan transformaciones.

Los modelos de excelencia de la gestión de calidad comparten el principio de liderazgo. La norma ISO 9001;2015 propone que la alta dirección debe demostrar liderazgo, compromiso y constante satisfacción del usuario (23). El modelo para la gestión de la calidad (24) destaca que el liderazgo debe ser ejercido con capacidad de visión. Los líderes establecen valores y principios éticos, y desarrollan una cultura y un sistema de gobierno de la organización que ofrece a sus grupos de interés una identidad. El modelo iberoamericano de excelencia en la gestión para las administraciones públicas menciona que los líderes promueven, motivan y estimulan a sus colaboradores, creando condiciones adecuadas para la mejora continua y la innovación, además de servir de modelo de referencia en comportamiento y rendimiento para los demás (25). Para el modelo Malcolm Baldrige, la gestión del liderazgo implica que la alta gerencia fije la dirección, se oriente hacia el usuario, determine valores claros y visibles y establezca altas expectativas de desempeño (26).

El cuadro 3 describe las teorías y características de liderazgo y su relevancia con SS-APS.

\section{DISCUSIÓN}

Las organizaciones de salud cuentan con poca experiencia para enfrentar a entornos cambiantes y competitivos. La gestión y liderazgo eficaz de los sistemas de salud basados en APS son fundamentales para entender y navegar a través de un sector cada vez más dinámico y complejo.

Los ministerios de salud deben planificar y gestionar estratégicamente de manera simultánea un sistema fragmentado y segmentado, sin descuidar otros elementos que irrumpen en ese entorno, tales como emergencias sanitarias y desastres. Esta es una carga demasiado pesada para la autoridad sanitaria nacional que tiene dificultades para atender a todo un sistema complejo y turbulento. En el futuro las organizaciones deberán ser más flexibles y los individuos aprenderán a depender más en sus redes interpersonales, en sus relaciones con el entorno mediato e inmediato y con la comunidad a la que se deben. Este planteamiento se ve reflejado en el interés de los sistemas de salud por promover la participación social y comunitaria, como componente fundamental de los SS-APS.

La gestión estratégica de sistemas de salud evidencia diferencias en sus subsistemas público y privado. La conducción debe comprender estas diferencias para alcanzar los consensos necesarios que permitan avanzar a SS-APS hacia la salud universal. La gestión estratégica es relevante, pues involucra desarrollar un pensamiento estratégico en el liderazgo y conducción del sistema que permita tener una perspectiva amplia desde donde surjan las decisiones estratégicas. Aunque el enfoque estratégico analítico sigue siendo útil para la gestión de los sistemas de salud, el enfoque emergente cobra cada vez más importancia, dado que el sector salud es un entorno cambiante. La gestión estratégica requiere gestionar impulsos estratégicos, la salud 
CUADRO 3. Liderazgo en sistemas de salud basados en APS hacia la salud universal

\section{Liderazgo Tipo/Clasificación \\ Lógica de acción (Rooke D, Torbert W.) \\ Descripción \\ Manera cómo el líder interpreta el entorno y reacciona a los desafíos. \\ Diplomático: Evita el conflicto. Sigue las normas. Evita tomar decisiones difíciles.}

Relevancia con sistemas de salud basados en APS
Experto: Gerencia por lógica, conocimiento y experiencia. Usa datos duros y evidencia para ganar consenso. Genera apoyo en el grupo que comparte la misma línea de trabajo.

Triunfador. Alcanza las metas estratégicas. Promueve el trabajo en equipo. Hace malabares con las tareas gerenciales, mientras responde a necesidades y demandas externas para alcanzar los objetivos. Idónea para un cargo gerencial. Pone en marcha las estrategias generadas desde niveles superiores

Estratega: Genera cambios organizacionales y personales. Altamente colaborativo/a, entrelaza visiones con iniciativas pragmáticas y oportunas; desafía normas preestablecidas.

Alquimista: Genera transformaciones sociales. Reinventa organizaciones de una manera históricamente significativa.

Uso de inteligencia emocional (Goleman D.)

Efectividad (Drucker P.)

Niveles de jerarquía (Collins J.)

Habilidades esenciales (Bennis W, Thomas R.)

Cualidades de liderazgo (Goffee R. Jones G.)
La inteligencia emocional es lo que distingue a los grandes líderes. Consta de cinco habilidades que el líder debe poseer: $\checkmark$ Autoconciencia

$\checkmark$ Autocontrol

$\checkmark$ Motivación

$\checkmark$ Empatía

$\checkmark$ Habilidades sociales

Ocho prácticas de los líderes efectivos:

Consigue el conocimiento requerido.

1. Investiga lo que requiere hacer.

2. Indaga lo que es lo mejor para la organización

Convierte el conocimiento en acción.

3. Desarrolla planes de acción.

4. Toma la responsabilidad por las acciones.

5. Toma responsabilidad en la comunicación.

6. Se enfoca en oportunidades, no en problemas

Asegura la rendición de cuentas.

7. Dirige reuniones productivas

8. Piensa y dice nosotros, no "yo"

Identifica cinco niveles de liderazgo: individuo capaz (nivel 1), líder efectivo (nivel 4) y finalmente el ejecutivo (nivel 5).

El nivel 5 de jerarquía de liderazgo: Involucra una voluntad fuerte con una gran dosis de humildad. Habilidades que permiten a los líderes aprender de la diversidad:

1. Embarca a otros en entendimientos comunes (shared meaning)

2. Integridad

3. Capacidad adaptativa

4. Voz distintiva y convincente

Cualidades necesarias para un liderazgo inspiracional:

1. Revela sus debilidades de manera selectiva

2. Se convierte en un sensor

3. Practica una fuerte y real empatía (tough empathy)

4. Se atreve a ser diferente.
Liderazgo de utilidad limitada. Puede consolidar conformación de equipos interinstitucionales en SS-APS.

Este liderazgo puede ser útil en un contexto local, donde la experticia es bien recibida. Los SS-APS prestan atención integral e integrada, I0 que implica un liderazgo que conozca y garantice su aplicación. Agrega evidencia y prestigio a la gestión.

La experiencia y resultados exitosos previos deben ser reconocidos y compartidos con sus pares, lo que permite su réplica en otras instancias del sistema. Es un liderazgo de actitud positiva que permite transitar en situaciones complejas y obtener los resultados deseados. Puente entre la teoría de las estrategias centrales y la realidad de la operatividad de la APS.

El estratega genera transformaciones en el corto y largo plazo y se ubica en el contexto de su realidad y la proyectada. Es el liderazgo necesario para la implementación del modelo de atención y la APS. EI estratega debe ser capaz de anticiparse al futuro y de adecuar la gestión a situaciones emergentes (i.e., epidemias, bloqueos políticos o económicos que impiden la llegada de insumos críticos, respuesta a desastres).

Lidera grandes cambios sociales. Es el liderazgo necesario cuando se busca cambiar el modelo de sistema nacional de salud para aumentar el acceso y la cobertura con miras a la Salud Universal.

El liderazgo de SS-APS requiere de inteligencia emocional para sortear los grandes retos y desafíos. Las habilidades descritas, en particular la empatía y las habilidades sociales, permiten generar alianzas y consensos que faciliten los cambios necesarios para avanzar hacia la salud universal. La motivación y las habilidades sociales destacan en un liderazgo en sistemas basados en APS dada su orientación a la justicia social, la participación y la intersectorialidad.

Este tipo de liderazgo permite identificar oportunidades entre los múltiples problemas a los que se enfrentan los sistemas de salud y permite el establecimiento de puentes que transformen el conocimiento en acción y en mecanismos de rendición de cuentas.

Se relaciona con el liderazgo del experto y triunfador (lógica de acción).

El liderazgo de SS-APS requiere voluntad para avanzar ante los obstáculos. El líder efectivo (nivel 4) y el líder ejecutivo (nivel 5) son los más requeridos en los sistemas de salud basados en APS.

La humildad que requiere el nivel 5 es una característica difícil de cumplir en el caso de Latinoamérica, pues puede confundirse con debilidad.

Estas habilidades permiten al líder avanzar en procesos de reforma hacia SS-APS, generando expectativas comunes, o adaptando el liderazgo a las condiciones imperantes, sin que en el proceso se pierda el principio de integridad o se deje de ser la voz convincente. La voz distintiva y convincente conlleva a una comunicación efectiva, mientras que la integridad dimensiona a los valores que permiten al líder enfrentar la falta de ética y la corrupción.

No existe un líder infalible y menos aun cuando involucra el liderazgo de un sistema tan complejo como el de salud. El aceptar las debilidades y asesorarse en ese campo es válido y denota la cualidad del líder. Los grandes líderes atravesaron por periodos de crisis o "crucibles" de los que salieron fortalecidos. La empatía hacia los colaboradores y colegas de otras entidades del sistema es clave para establecer consensos y alianzas en sistemas fragmentados. 
CUADRO 3. Liderazgo en sistemas de salud basados en APS hacia la salud universal (continúa)

\begin{tabular}{|c|c|c|}
\hline $\begin{array}{l}\text { Liderazgo } \\
\text { Tipo/Clasificación }\end{array}$ & Descripción & Relevancia con sistemas de salud basados en APS \\
\hline $\begin{array}{l}\text { Autenticidad } \\
\text { (George Bill et al.) }\end{array}$ & $\begin{array}{l}\text { Para alcanzar un liderazgo auténtico se requiere: } \\
\text { 1. Entender y aprender de su historia (de vida) } \\
\text { 2. Conocer su "yo" interior (self-awareness) } \\
\text { 3. Practicar sus valores y principios } \\
\text { 4. Mantener un balance entre motivaciones intrínsecas y } \\
\text { extrínsecas }\end{array}$ & $\begin{array}{l}\text { Se relaciona con la inteligencia emocional y las habilidades } \\
\text { esenciales que debe poseer un líder. Los factores intrínsecos como la } \\
\text { autoconciencia, el autocontrol, valores y principios innatos son pilares } \\
\text { que el líder estratega o alquimista requiere en procesos de mediano } \\
\text { y largo plazo para el desarrollo de SS-APS. Estos elementos son de } \\
\text { gran valor cuando el sistema enfrenta un debilitamiento de la autoridad } \\
\text { sanitaria. }\end{array}$ \\
\hline
\end{tabular}

Protección: Permite cierta presión externa que sea

manejable

Orientación: Desafía roles actuales y resiste presión para

definir nuevos roles

Manejo de conflictos: Expone o deja emerger el conflicto

Modula normas: desafía normas inadecuadas.

$\begin{array}{ll}\text { Liderar no es gerenciar } & \text { LíDER } \\ \text { (Kotter) } & \checkmark \text { Establece una dirección } \\ & \checkmark \text { Alinea actores } \\ \checkmark & \text { Motiva } \\ & \text { GERENTE } \\ \checkmark & \text { Planifica y presupuesta } \\ \checkmark & \text { Organiza } \\ & \checkmark \text { Controla y resuelve problemas } \\ \text { Liderazgo como modelo } & - \text { Modelos de excelencia: } \\ \text { de calidad } & \text { 1. ISO 9001 } \\ & \text { 2. EFQM } \\ \text { 3. Modelo Iberoamericano de excelencia en la gestión para } & \text { las administraciones públicas } \\ & \text { 4. Modelo Malcolm Baldrige }\end{array}$

Los sistemas de salud basados en APS requieren de líderes más que de gerentes. Los sistemas fragmentados requieren de expectativas comunes que busquen alinear actores hacia un mismo objetivo.

Establecen criterios claves para un liderazgo comprometido, visionario e inspirador, que motive a la excelencia guiada por el ejemplo, con valores y principios éticos, metas claras y alcanzables comunicadas a lo largo de la organización, con espacios para la innovación y la mejora continua que permita la creación de una identidad organizacional.

Los sistemas basados en APS requieren de un liderazgo enfocado en la provisión de servicios de salud integrales con gestión de calidad, dado que son los elementos más visibles del sistema.

Los modelos expuestos comparten elementos con otras teorías de liderazgo expuestas. Los modelos de calidad aportan con otros criterios tales como el principio de identidad organizacional, considerado necesario para generar un sentido de apropiación de los sistemas de salud por parte de los usuarios externos e internos. El liderazgo innovativo es más difícil de implementar en el subsistema público por la rigidez de las normas y procedimientos.

La mejora continua, como principio de calidad, es un criterio de liderazgo que comparte con otras teorías y que es fundamental para Ios procesos inherentes a SS-APS y que permitiría cierto nivel de innovación.

Fuente: los autores

Fuente: los autores
SS-APS, sistemas de salud basados en atención primaria de la salud

universal debe convertirse en ese impulso y son los líderes los llamados a generar y mantener la inercia para alcanzar el acceso y la cobertura universal.

El líder de un SS-APS debe tener la flexibilidad de acomodar diferentes estilos de liderazgo. En un sistema con servicios de salud fragmentados y segmentados el liderazgo también está dividido, lo que genera dificultades en la búsqueda de consensos entre los actores del sistema, aunque tampoco significa que tiene que ser único. Históricamente, el liderazgo del sistema de salud ha recaído en la autoridad sanitaria nacional, el ministro/a de salud, aunque existen otros liderazgos tales como el de un superintendente de salud o de una directora de seguridad social, que pueden llegar a tener más influencia en las decisiones sanitarias. Es preciso definir esas dinámicas y el tipo de liderazgo que requieren los países para lograr sistemas de salud adaptativos, resilientes, que les permita avanzar hacia la salud universal.

La visión hacia un sistema de salud único integral e integrado basado en APS no debe provenir solo de un liderazgo formal y único que puede estar sesgado o equivocado como lo afirma Heifetz (10), sino también de "liderazgos de base" que sustenten la visión y generen el impulso para avanzar en una misma dirección. Ese tipo de liderazgo obliga a una alternabilidad entre permanecer "en el balcón" mirando el escenario desde una posición estratégica, y bajar al campo de acción para empaparse de la cotidianidad. Además, esta "democratización" del liderazgo sería una alternativa para neutralizar un liderazgo erróneo. 
Consideramos que para una reforma de salud se requiere las lógicas de acción del estratega, pero si se busca la transformación del sistema nacional de salud es el alquimista el llamado a liderar ese tipo de cambio. Sin embargo, el liderazgo en SS-APS requiere en determinado momento de rasgos o características de otras lógicas de acción. La lógica del experto o del triunfador pueden tener su espacio al tender puentes entre la teoría de las estrategias centrales y la realidad de la operatividad. En situaciones de emergencia sanitaria la lógica de acción del oportunista puede ser útil mientras dure la contingencia, a la que podría sumarse la del diplomático cuando se busca alcanzar consensos. El líder puede ignorar ciertas normas en un momento dado, como lo hace el individualista o que se preste a cumplir tareas gerenciales más compatibles con la lógica del triunfador. El principal riesgo es que la conducción del sistema quede estancada en un solo tipo de liderazgo, impasible al cambio y a las circunstancias del entorno.

La evidencia consultada y eminentemente anglosajona genera las siguientes preguntas: ¿Estas teorías y categorías de liderazgo aplican a nuestros países? ¿Es el liderazgo orientado por características socioculturales? La respuesta a ambas es que sí. El liderazgo tiene rasgos comunes en todas partes, pero es claro que existen características propias que varían en diferentes sociedades y culturas. El liderazgo de nivel 5 que propone Collins (humildad y voluntad), en determinados contextos latinoamericanos podría ser percibido como débil y poco motivante. En Latinoamérica se mantiene el influjo del caudillismo y persiste la imagen del líder fuerte, extrovertido y carismático. Los sistemas de salud no son ajenos a esa realidad y su conducción deberá ajustarse a las circunstancias.

La intuición mantiene un espacio en los sistemas de salud, dada la limitada disponibilidad de datos de desempeño e información de inteligencia que permitan la toma de decisiones estratégicas basadas en evidencia. Por otro lado, los sistemas de salud están sujetos a mandatos y obligaciones que limitan la autonomía y flexibilidad en la toma de decisiones, dejando poco espacio para que el liderazgo base sus decisiones en intuiciones. La intuición se mantiene como una herramienta para la toma de decisiones estratégicas porque brinda un sentido estratégico, puede tener alguna utilidad cuando el objetivo es explorar nuevas estrategias y tecnologías, cuando el costo personal y organizacional del fracaso puede ser absorbido, cuando las restricciones de tiempo y recursos evitan contar con el conocimiento necesario, o porque "aún conserva cierto encanto", (17) aunque su utilidad en SS-APS es cuestionada.

Los modelos de calidad analizados enfatizan la importancia del liderazgo en los sistemas de salud, en particular en la red de servicios. Los criterios que establecen son similares a las teorías de liderazgo antes descritas, aunque destacan la innovación y la identidad organizacional como aportes de estos modelos. En particular, la identidad de los SS-APS es un componente fundamental, considerando el pobre apropiamiento que tiene la población de sus sistemas públicos, especialmente cuando son financiados por el presupuesto general. Por otro lado, el liderazgo innovativo es más difícil de implementar en un subsistema público por la rigidez de las normas y procedimientos. La mejora continua, como principio de calidad, es un criterio de liderazgo que comparte con otras teorías y que es fundamental para los procesos inherentes a sistemas de salud basados en APS y que permitiría cierto nivel de innovación.
FIGURA 1. Principales características de liderazgo en sistemas de salud basados en APS

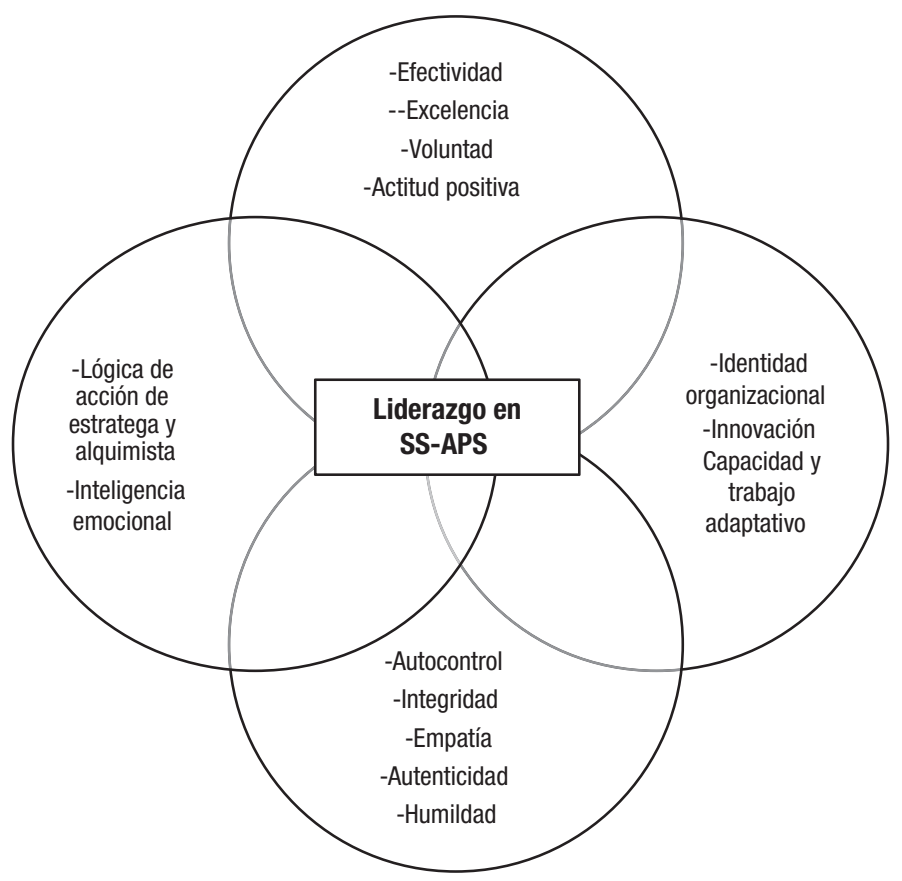

Fuente: los autores

SS-APS, sistemas de salud basados en atención primaria de la salud

En conclusión, Latinoamérica debe caminar hacia SS-APS altamente participativos, con líderes que deben aprender a liderar, descubriendo sus propios eventos transformadores, desarrollando inteligencia emocional, buscando la calidad, combinando varios tipos de liderazgo en función de la situación, utilizando las lógicas de acción del diplomático cuando se requiere negociar o coordinar con socios externos, siendo estrategas para liderar a las entidades del sistema en su transformación a organizaciones resilientes, siendo alquimistas para lograr transformaciones del modelo de sistema de salud, con la voluntad y humildad de quien no trabaja para una agenda personal, sino para alcanzar la salud universal (figura 1).

Contribuciones de los autores. EBP definió el objetivo y elaboró el diseño del estudio. EBP, JMS, GR contribuyeron con la búsqueda bibliográfica. JMS, GR realizaron la matriz principal de análisis, resultados y discusión del artículo. Todos los autores escribieron, revisaron y aprobaron la versión final del manuscrito.

Agradecimientos. Los autores reconocen y agradecen a los líderes y responsables de salud en todos los niveles en las Américas, sobre todo a aquellos que desde el anonimato hacen grandes esfuerzos trabajando y motivando día a día a sus equipos para lograr la salud universal.

\section{Conflicto de intereses. Ninguno declarado.}

Declaración. Las opiniones expresadas en este manuscrito son únicamente responsabilidad de los autores y no reflejan necesariamente los criterios ni la política de la $R P S P / P A J P H$ o la Organización Panamericana de la Salud. 


\section{REFERENCIAS}

1. Organización Panamericana de la Salud. Salud Universal en el Siglo XXI: 40 años de Alma-Ata. Washington, D.C: OPS/OMS; 2019.

2. The Tallinn Charter: Health Systems for Health and Wealth. Tallinn, Estonia, 25-27 June 2008.: WHO. Regional Office for Europe; 2008. Disponible en: http://www.euro.who.int/_data/assets/pdf_ file/0008/88613/E91438.pdf. Acceso el día 10 de septiembre de 2019.

3. Macinko J, Montenegro H, Nebot Adell C, Etienne C, Grupo de Trabajo de Atención Primaria de Salud de la Organización Panamericana de la Salud. La renovación de la atención primaria de salud en las Américas. Rev Panam Salud Publica. 2007;21 $(2 / 3): 73-84$

4. Giovanella L, editor. Atención Primaria de Salud en Suramérica. Río de Janeiro: Instituto Suramericano de Gobierno en Salud. Unión de Naciones Sudamericanas; 2015. Disponible en: https:/ / redeaps. org.br/wpcontent/uploads/2019/07/livro_atencao_primaria_ de_saude_2015_esp-2-1.pdf Acceso el día 18 de septiembre de 2019.

5. Ruiz F, Zapata T, Garavito L. Colombian health care system: results on equity for five health dimensions. Rev Panam Salud Publica. 2013;33(2):107-15.

6. Báscolo E, Houghton N, Del Riego A. Lógicas de transformación de los sistemas de salud en América Latina y resultado en acceso y cobertura de salud. ). Rev Panam Salud Publica. 2018;42:e126. https://doi.org/10.26633/RPSP.2018.126

7. Popay J, Roberts H, et al. Guidance on the Conduct of Narrative Synthesis in Systematic Reviews. Economic and Social Research Council; 2006.

8. Kotter JP. What leaders really do. Harv Bus Rev. 1990 May-Jun; 68(3):103-11.

9. Ginter P, Duncan W, Swayne L. The nature of strategic management. In: Strategic Management of health care organizations. 7th ed. San Francisco.: Jossey-Bass; 2013. p. 1-32.

10. Heifetz RA, Laurie DL. The work of leadership. Harv Bus Rev. 1997 Jan-Feb;75(1):124-34.

11. Zuckerman A. Creating a vision for the twenty-first century healthcare organization. J Healthc Manag. 2000;45(5):295.

12. Drucker P. What Makes an Effective Executive. Har Bus Rev. 2001:18.

13. Poister T, Pitts D, Edwards L. Strategic Management Research in the Public Sector: A Review, Synthesis, and Future Directions. Am Rev Public Adm. 2010;40:522-545.

14. Nutt PC. Comparing Public and Private Sector Decision-Making Practices. J Public Adm Res Theory. 2005;6:289-318.
15. Harris M. Strategic Planning in an International Nongovernmental Development Organization: The Creation of a Meta-Identity. Admin Soc. 2011;43(2):216-247.

16. Kellermanns FW, Walter J, et al. To agree or not to agree? A meta-analytical review of strategic consensus and organizational performance. J Bus Res. 2011;64:126-133.

17. Miller C, Ireland D. Intuition in strategic decision making: Friend or foe in the fast-paced 21st century? Acad Manag Perspect. 2005;19(1):19-30.

18. Secretaría de Salud de México. Modelo de gestión de calidad en salud 2016. México: Dirección General de Calidad y Educación en Salud; 2017.

19. Bennis WG, Thomas RJ. Crucibles of leadership. Harv Bus Rev. 2002 Sep;80(9):39,45, 124.

20. Collins J. Level 5 leadership. The triumph of humility and fierce resolve. Harv Bus Rev. 2001 Jan;79(1):66,76, 175.

21. Goleman D. What makes a leader? Harv Bus Rev. 1998 Nov-Dec; 76(6):93-102.

22. Rooke D, Torbert WR. 7 Transformations of Leadership. Harv Bus Rev. 2005 Apr;83(4):66,76, 133

23. Cruz F, López A, Ruíz C. Sistema de Gestión ISO 9001-2015: Técnicas y herramientas de ingeniería de calidad para su implementación. Rev Ingeniería Investigación y Desarrollo. 2017;17(1).

24. European Foundation of Quality Management. EFQM Management Document. 2018. Disponible en: https://www.efqm.org/ index.php/download/efqm-management-document-_guideline/. Acceso el 19 de septiembre de 2019.

25. FUNDIBEQ. Modelo Iberoamericano de excelencia en la gestión para las administraciones públicas. 2019. Disponible en: https:// www.fundibeq.org/images/pdf/Modelo_Iberoamericano_V2019_ AP.pdf). Acceso el día 18 de septiembre de 2019.

26. Baldrige National Quality Program. Modelo de excelencia en la gestión. 2006. Disponible en: http://www.praxis.com.pe/portal/ sites/default/files/m_baldrige_2006.pdf: 2006. Acceso el día 18 de septiembre de 2019.

Manuscrito recibido el 25 de septiembre de 2019. Aceptado para su publicación, tras revisión, el 26 de mayo de 2020. 


\section{Leadership and strategic management in health systems based on primary health care}

ABSTRACT Objectives. To identify and analyze various types of leadership and management that could contribute to the strengthening of health systems based on primary health care $(\mathrm{PHC})$.

Methods. A structured review of the literature on management and leadership applicable to PHC-based health systems was conducted. Of the 19 articles identified, relevant details were extracted using a standardized methodology.

Results. PHC-based health systems should establish optimal management practices to achieve quality, efficiency, and effectiveness. Strategic planning and management facilitate the development of a common identity, although some organizations are overmanaged and poorly led. Universal health is a strategic driver that requires transformational leadership to move toward PHC-based systems. Leadership in these systems must focus on identifying adaptive challenges, linking staff and protecting grassroots leadership, as well as adapting to the times and combining the logic of action of the strategist to achieve a sector transformation. The quality models establish leadership criteria similar to other theories, emphasizing innovation and organizational identity.

Conclusions. Latin America must move towards health systems based on PHC, with leaders with emotional intelligence who discover their own transformative events, seek quality, and combine various types of leadership. They must be strategists to lead the system in its transition to resilient organizations, and "alchemists" to achieve true transformations in health, with the willingness and humility of those who work to achieve universal health.

Keywords Health management; leadership; health systems; primary health care; Américas. 